\title{
La crisis de la TDT local pública en España: el caso de Cataluña
}

\section{The Crisis of Public Local Digital Terrestrial Television in Spain: The case of Catalonia}

\author{
Dr. Josep Àngel Guimerá i Orts - Profesor del Departamento de Comunicación \\ Audiovisual y Publicidad I - Universitat Autònoma de Barcelona, UAB - \\ JosepAngel.Guimera@uab.cat
}

Lic. Federica Alborch Gil - Doctoranda del Departamento de Medios, Comunicación y Cultura - Universitat Autònoma de Barcelona, UAB -

MariaFederica.Alborch@campus.uab.cat

Resumen: El artículo describe y analiza el estado de la televisión digital terrestre local (TDT-L) pública en Cataluña justo después del apagón analógico de abril de 2010. Los resultados muestran que sólo 12 de los 37 canales públicos previstos estaban emitiendo y que sólo cinco más podían hacerlo a medio plazo. Estos datos indican que la televisión pública local vive una crisis en el contexto de la migración digital, ya que estos canales sufren graves limitaciones para nacer y el apagón implica la desaparición de emisoras analógicas históricas. El artículo se basa en una investigación cualitativa en el transcurso de la cual se ha entrevistado en profundidad a representantes de los 37 canales previstos. Los objetivos del artículo son, por una parte, describir el modelo de TDT-L vigente y su estado de implantación en Cataluña en mayo de 2010. Por otra parte, aportar elementos interpretativos que expliquen la situación de crisis identificada. Finalmente, aportar conocimiento que permita formular hipótesis sobre el estado del sector en el resto de España. Los resultados ponen de manifiesto que los problemas de la TDT-L pública tienen su origen en las políticas de implantación digital de los gobiernos español y catalán, que no han tenido en cuenta la realidad analógica preexistente -sobre todo el primero. En este sentido, el caso catalán aporta indicios sobre la situación en el conjunto de España.

Palabras clave: Política de la comunicación; medios de comunicación de masas; TDT; televisión local; televisión pública; Cataluña.

Abstract: The article describes and analyses the state of public local digital terrestrial television (L-DTT) in Catalonia just after the analogue switch-off in April 2010. The results show that only 12 of the 37 anticipated public television channels were broadcasting, and only five more were expected to do so in the medium term. These data indicate that public local television was experiencing a crisis in the context of the digital switchover process: while some digital programmes were finding it hard to get off the ground due to severe 
limitations, other historical analogue stations were disappearing. The article is grounded on qualitative research based on in-depth interviews with representatives of the 37 aforementioned channels. The objectives of the paper are: to describe the L-DTT model and its roll-out status in Catalonia as at May 2010; to provide interpretative elements to explain the crisis identified; and to provide insights for the formulation of hypotheses about the state of the sector in the rest of Spain. The results suggest that the problems of public L-DTT stem from the digital roll-out policies of the Government of Spain and the Government of Catalonia, neither of which - especially the first - seems to have taken into account the preexisting analogue reality. In this respect, the Catalan case offers signs and evidence that can be extrapolated to the situation in Spain as a whole.

Keywords: communication policy; mass media; DTT; local television; public television; Catalonia.

Sumario: 1. Introducción. 2. Metodología 3. Estructura básica de la TDT-L pública en Cataluña (2010). 4. Estado de los programas públicos de TDT-L en Cataluña (mayo 2010). 5. La demarcación y el modelo de TDT-L público: dos elementos clave. 6. El desarrollo de la TDT-L pública: elementos interpretativos. 6.1. Los consorcios en funcionamiento: rasgos característicos. 6.1.1. Municipios medianos o grandes con capacidad de liderazgo. 6.1.2. Televisiones analógicas profesionalizadas en la base del proyecto. 6.1.3. La vinculación con proyectos estratégicos. 6.2. Los consorcios sin televisión: una casuística compleja. 6.2.1. El factor económico: la principal razón. 6.2.2. La falta de liderazgo claro: hacia el estancamiento del consorcio. 6.2.3. El escaso interés por la televisión. 6.2.4. La crisis económica como agravante. 6.2.5. Un exceso de reservas para la iniciativa pública. 7. Conclusiones. 8. Fuentes consultadas. 9. Notas.

Summary: 1. Introduction. 2. Methodology 3. Basic structure of public L-DTT in Catalonia (2010). 4. State of public L-DTT programmes in Catalonia (May 2010). 5. Demarcation and the public L-DTT model: two key elements. 6. Development of the L-DTT service: interpretative elements. 6.1. Consortiums in operation: characteristic traits. 6.1.1. Medium and large municipalities with leadership potential. 6.1.2. Professionalised analogue television stations at the base of the project. 6.1.3. The link with strategic projects. 6.2. Consortiums without television: complex causes. 6.2.1. The financial factor: the main cause. 6.2.2. A lack of clear leadership: bringing a consortium to a standstill. 6.2.3. A lack of interest in television. 6.2.4. The recession as an aggravating factor. 6.2.5. Too many programmes set aside for the public initiative. 7. Conclusions. 8. References. 9. Notes

Title, abstract, keywords and contents translated into English by Silvia Solá, Servei de Llengües de la Universitat Autònoma de Barcelona (UAB), and Steven Norris. Article translated into English by Steven Norris. 


\section{Introducción}

A mediados de 2010, la televisión local pública catalana estaba atravesando una crisis de dimensiones notables. En abril de ese año, justo después de completarse el apagón analógico, sólo emitían 12 televisiones digitales terrestres locales (TDT-L) públicas, menos de un tercio de las 37 previstas por el Gobierno catalán. A medio plazo, sólo cinco programas digitales públicos más podían llegar a emitir.

Estas cifras chocan con las 54 estaciones analógicas municipales en funcionamiento en 2006, año en que la Generalitat de Cataluña otorgó las concesiones de TDT-L (Guimerà et al. 2009). Así mismo, el peso de la iniciativa pública en el conjunto de la televisión local (TVL) del Principado ha bajado sensiblemente con la introducción de la TDT. Si en 2006, esas 54 estaciones representaban el $43 \%$ de las 128 TVL en funcionamiento, las 12 digitales sólo suponían el 23,5\% de las 51 emisoras de TDT-L que emitían en mayo de 2010. Estas cifras apuntan al fin de la hegemonía de la televisión pública en Cataluña, que a principios de los años dos mil era más de la mitad del sector (OCL, 2005).

La situación descrita pone de manifiesto que la implantación de la TDT-L en España ha comportado una fuerte reestructuración de la televisión local. De hecho, diversas investigaciones han puesto de manifiesto que la televisión local digital es un fenómeno sustancialmente diferente de la TVL analógica (Guimerà, 2006; Fernández et al., 2007; Corominas, 2009; Ortega, 2009; Marzal y Casero, 2008). Esto se debe a que la transición digital de esta modalidad televisiva no se ha hecho teniendo en cuenta la realidad analógica preexistente, sino más bien todo lo contrario: la TDT-L, que trae consigo la plena regulación de la televisión local en España ${ }^{1}$, introduce un modelo digital que poco tiene que ver con el predominante en la era analógica (Fernández et al., 2007; Ortega, 2009).

El principal motivo de esta reordenación es la decisión del Gobierno central de organizar la TDT-L a partir de demarcaciones, formadas en su gran mayoría por más de un municipio (hay casos que agrupan casi treinta). Esta organización rompe con la estrecha relación que la TVL analógica había mantenido con el municipio, "unidad básica de organización" del sector hasta la llegada de la televisión digital (Corominas, 2009: 7).

Los medios de titularidad pública son los que más han sufrido las consecuencias de ese cambio (Guimerà, 2006; Guimerà, Sanmartín y Alborch, 2008; CAC, 2009). La razón es que las políticas públicas reservaron para la titularidad pública un máximo de dos programas digitales (equivalente a los antiguos canales analógicos) de los cuatro disponibles en cada una de las 275 demarcaciones ${ }^{2}$ en que se ha organizado la TDT-L en España. De estas, sólo 10 están formadas por un solo municipio (Fernández et al., 2007). La combinación de ambos elementos ha provocado que, en la mayoría de los casos, los ayuntamientos interesados en gestionar el servicio deban ponerse de acuerdo para explotar conjuntamente uno de esos programas. 
La consecuencia es la aparición de un modelo de gestión completamente nuevo en la televisión pública local, ya que en la era analógica cada ayuntamiento podía disponer de su propio canal (Guimerà, 2006; Corominas, 2009). Son precisamente estos nuevos canales de gestión conjunta los que han causado más problemas en la implantación de la TDT en Cataluña: de los 25 que no emitían en mayo de 2010, 24 correspondían a grupos de municipios que no consiguieron ponerse de acuerdo. A su vez, estos canales son uno de los fenómenos más novedosos, y a la vez desconocidos, que la TDT ha introducido en el ámbito local (Marzal y Casero, 2008: 90).

Sin duda alguna, a raíz del apagón analógico de abril de 2010 se ha gestado en España un nuevo sector público de TDT-L que está sufriendo notables problemas y limitaciones en sus primeros pasos. El presente artículo describe y analiza este proceso centrándose en el caso de Cataluña. La experiencia de esta comunidad autónoma es especialmente significativa porque era, junto con Andalucía, la que más emisoras analógicas municipales tenía en los años previos a la transición digital (Badillo, 2005; Guimerà, 2006). Además, el Gobierno autonómico hizo una fuerte apuesta por el sector público reservando para éste 37 de los 96 programas de que disponía Cataluña. En términos relativos, ha sido la comunidad que más espacio ha reservado para el servicio público $(38,5 \%)$, seguida a cierta distancia por Canarias (30,5\%) (Ortega, 2009).

Es en este contexto que resulta pertinente plantearse por qué razones está costando tanto poner en marcha la TDT-L pública en el estado español tomando como caso de estudio un territorio con un sector amplio y dinámico con casi treinta años de experiencia -la primera televisión municipal española nació en Cataluña en 1983 (Guimerà, 2006). Así, el propósito de este artículo es describir el estado (crítico) en que se encentraba la TDT-L pública catalana en los momentos posteriores al apagón analógico y aportar elementos interpretativos que ayuden a entender esa situación. Así, se analizan tanto los elementos que dificultan el arranque como también las razones que ayudan a entender los casos que han conseguido ponerse en marcha.

\section{Metodología}

El artículo se basa en los resultados obtenidos en una investigación sobre el estado de la implantación de la TDT en Cataluña encargado por el Consell de l'Audiovisual de Catalunya $(\mathrm{CAC})^{3}$ al primer autor del presente artículo. El trabajo de campo, llevado a cabo entre junio de 2008 y mayo de 2009, se basó en la visita de las instalaciones de los 83 concesionarios de TDT-L existentes en ese periodo (46 privados y 37 públicos) y en entrevistas a sus responsables.

En el caso de los prestadores públicos, los entrevistados fueron los directores de la televisión, en aquellos casos que los tuvieran, o el alcalde del municipio que debería liderar el consorcio para la gestión conjunta del canal, en aquellos casos en que la televisión todavía no había 
arrancando. En algunos casos, se delegó en el concejal competente, habitualmente el de cultura (CAC, 2009). Estas entrevistas son la fuente clave sobre la que se basa este artículo.

Dada la complejidad del fenómeno a estudiar, las conversaciones se plantearon como entrevistas abiertas semiestructuradas. En ellas se preguntaba a los entrevistados sobre el estado de implantación de la TDT en su demarcación; el estado de desarrollo de su proyecto y los eventuales factores que influyeran en el proceso. A la luz de los resultados obtenidos en una investigación exploratoria previa (Guimerà, Sanmartín y Alborch, 2008), se tenía constancia que el proceso de gestación de los nuevos canales públicos no estaba resultando nada fácil y que los factores que influían eran numerosos e interrelacionados entre sí.

Plantear una investigación de matriz hipotético - deductivo se revelaba poco apropiada, ya que se corría el riesgo de excluir algún elemento clave en el momento de diseñar las hipótesis (Soriano, 2007). Es por esto que en la investigación se optó por un diseño cualitativo que permitiera, potencialmente, captar toda la complejidad de un objeto de estudio nuevo y poco estudiado (la TDT-L pública) inmerso en un proceso en marcha (la transición digital) en el momento de la recogida de datos (Marzal y Casero, 2008). Los resultados obtenidos en las entrevistas realizadas entre 2008 y 2009 se han complementado con la documentación facilitada por las administraciones locales (presupuestos, parrillas programáticas, planes de inversión) durante el trabajo de campo.

Estos datos se han actualizado hasta mayo de 2010 a través de consultas puntuales al CAC y a los entes públicos locales. Así mismo, se han consultado numerosas fuentes hemerográficas que han permitido seguir la evolución del sector hasta mayo de 2010. Toda esta documentación se ha interpretado y contextualizado a la luz de estudios pioneros sobre la transición digital de la televisión local en España (Fernández et al., 2007; Guimerà, 2006; Ortega, 2009; Guimerà et al., 2009; Corominas, 2009).

El valor de este artículo va más allá de lo estrictamente referente a Cataluña. Se basa en la primera investigación que analiza en profundidad el conjunto del sector público local digital de una comunidad autónoma. Así mismo, es la primera investigación sobre estructura de la comunicación de masas de la que se tiene constancia en España que haya considerado la totalidad del universo a estudiar y que, además, haya hecho una aproximación cualitativa a éste.

De esta manera, el artículo aporta información que puede ayudar a estudiar el fenómeno en otras zonas de España, ya que permite la formulación de hipótesis al respecto. Desde esta perspectiva, la riqueza de la experiencia catalana la convierte en un escenario de máximo interés para el estudio de la TDT-L pública en España en el contexto de la migración digital.

\section{Estructura básica de la TDT-L pública en Cataluña (2010)}

En mayo de 2010, justo un mes después del apagón analógico, Cataluña contaba con 37 programas de TDT-L reservados para la administración pública local (ver la Tabla 1). Esta 
cifra surgió de las decisiones políticas tomadas tanto por el Gobierno español como por el catalán durante los años 2004 y 2005.

Las políticas estatales determinaron que la implantación de la TDT-L debía llevarse a cabo a partir de una nueva "unidad básica de organización" denominada demarcación (Corominas, 2009: 7). El Plan Técnico Nacional de la Televisión Digital Local (PTNTDL) aprobado en $2004^{4}$, dividió Cataluña en 21 demarcaciones. Cada una de ellas consta de un ámbito de cobertura formado por agrupaciones de municipios que van desde los tres de Vielha e Mijaran hasta los 28 de Cornellá (Ver la Tabla 1).

Ninguno, pues, corresponde a uno solo y el total de municipios planificados es de 240. A su vez, todas las demarcaciones catalanas cuentan con un canal múltiplex (MUX), aunque hay tres que tienen dos (Barcelona, Cornellà de Llobregat y Sabadell). Por lo tanto, Cataluña cuenta con 24 MUX. Cada uno de ellos cuenta con cuatro programas digitales (equivalentes a les antiguos canales analógicos), de manera que se pueden crear hasta 96 TDT-L.

Es a partir de esta organización en demarcaciones que el Gobierno central fijó cómo se debía repartir el espacio radioeléctrico. La ley 41/1995, de 22 de diciembre, de regulación de la televisión local por ondas (BOE 309, de 27 de diciembre) modificada en $2005^{5}$ determina que al menos uno de los programas de cada múltiplex se debe reservar a la gestión pública, aunque las comunidades autónomas podrán reservar hasta dos en función de sus necesidades. En las demarcaciones con dos MUX, se puede llegar a reservar uno entero para los consistorios y otro para los privados. El resto del espacio disponible se reserva para la gestión privada.

De acuerdo con esto, y después de consultar a los ayuntamientos, el Gobierno catalán decidió reservar 37 programas para la gestión directa por parte de los municipios y 59 para la gestión privada. De los 37 públicos, cuatro correspondían a un ayuntamiento en solitario (Badalona, Barcelona, L'Hospitalet de Llobregat y Reus), mientras que los otros 33 tenían que ser gestionados por diferentes agrupaciones de municipios (Ver tabla 1).

Tal y como ha puesto de manifiesto la investigación académica, una de las características de todas las demarcaciones catalanas es que están integradas por conjuntos de municipios que, "como tales, no constituyen una unidad administrativa o política reconocida", por lo que se convierten en un fenómeno nuevo y exclusivo de la TDT-L (Corominas, 2009: 16). Así, el proceso para gestionar el programa debe comenzar con negociaciones muy básicas.

De hecho, hay que tener en cuenta que la Generalitat de Cataluña decidió, en algunos casos, reservar más de un programa para la gestión pública ante la avalancha de peticiones que

recibió por parte de los consistorios: de los más de 200 municipios planificados, sólo uno dijo que no estaban interesados en hacer televisión. Con su decisión, el Gobierno quería facilitar los acuerdos entre los consistorios implicados (Guimerà, 2006). 
Tabla 1. Distribución de los programas públicos de TDT-L (2010)

\begin{tabular}{|c|c|c|c|}
\hline Demarcación & $\begin{array}{l}\text { Referencia } \\
\text { MUX }\end{array}$ & $\begin{array}{l}\text { Programas } \\
\text { públicos }\end{array}$ & Municipios concesionarios \\
\hline \multirow{4}{*}{ Barcelona } & \multirow{4}{*}{ TL01B } & \multirow{4}{*}{4} & 1. Barcelona \\
\hline & & & 2. L'Hospitalet de Llobregat \\
\hline & & & 3. Badalona \\
\hline & & & 4. Santa Coloma de Gramenet, Sant Adrià de Besòs \\
\hline \multirow{4}{*}{$\begin{array}{l}\text { Cornellà de } \\
\text { Llobregat }\end{array}$} & \multirow[b]{4}{*}{ TL11B } & \multirow[b]{4}{*}{4} & 1.Cornellà de Ll., Esplugues de Ll., S. Joan Despí i S.Just Desvern \\
\hline & & & $\begin{array}{l}\text { 2. S.Vicenç dels Horts, Molins de Rei, Vallirana, S.Feliu de Llobregat, Pallejà, } \\
\text { Cervelló, Sta.Coloma de Cervelló, el Papiol, la Palma de Cervelló }\end{array}$ \\
\hline & & & $\begin{array}{l}\text { 3.Martorell, S.Andreu de la Barca, Olesa de Montserrat, Esparreguera, Abrera, } \\
\text { S.Esteve Sesrovires, Collbató, Castellví de Rosanes }\end{array}$ \\
\hline & & & 4.S.Boi de Llobregat, el Prat de Ll., Viladecans, Castelldefels, Gavà, Begues \\
\hline \multirow[b]{3}{*}{ Sabadell } & \multirow[b]{3}{*}{ TL12B } & \multirow[b]{3}{*}{3} & $\begin{array}{l}\text { 1.Sabadell, Barberà del Vallès, Castellar del Vallès, Palau-solità i Plegamans, } \\
\text { Sentmenat i Polinyà }\end{array}$ \\
\hline & & & 2.Terrassa, Matadepera i Viladecavalls \\
\hline & & & $\begin{array}{l}\text { 3.Rubí, S.Cugat del Vallès, Cerdanyola del Vallès, Ripollet, Montcada i Reixac, } \\
\text { Sta.Perpètua de Mogoda, S. Quirze del Vallès, Castellbisbal. }\end{array}$ \\
\hline \multirow[b]{2}{*}{ Granollers } & \multirow[b]{2}{*}{ TL02B } & \multirow[b]{2}{*}{2} & $\begin{array}{l}\text { 1.Granollers, Franqueses del V., Cardedeu, Caldes de Montbui, S. Celoni, Canovelles, } \\
\text { la Garriga, Lliçà d'Amunt, la Roca del V., Llinars del V., Sta.Maria de Palautordera, } \\
\text { l'Ametlla del Vallès, Bigues i Riells, Lliçà de Vall, S.Feliu de Codines, Sta.Eulàlia de } \\
\text { Ronçana, S. Antoni de Vilamajor, S.Pere de Vilamajor }\end{array}$ \\
\hline & & & $\begin{array}{l}\text { 2.Mollet del Vallès, Parets del Vallès, Montornès del V., la Llagosta, Montmeló, } \\
\text { S.Fost de Campsentelles, Martorelles, Vallromanes }\end{array}$ \\
\hline \multirow[b]{2}{*}{ Mataró } & \multirow[b]{2}{*}{ TL06B } & \multirow[b]{2}{*}{2} & $\begin{array}{l}\text { 1.Mataró, Premià de Mar, el Masnou, Vilassar de Mar, Argentona, Premià de Dalt, } \\
\text { Alella, Montgat, Sant Andreu de Llavaneres, Vilassar de Dalt, Tiana, Teià, Cabrils, } \\
\text { Sant Vicenç de Montalt, Cabrera de Mar. }\end{array}$ \\
\hline & & & $\begin{array}{l}\text { 2.Pineda de Mar, Malgrat de Mar, Calella, Arenys de Mar, Canet de Mar, Tordera, } \\
\text { Arenys de Munt, Palafolls, Sant Pol de Mar }\end{array}$ \\
\hline \multirow[b]{2}{*}{$\begin{array}{l}\text { Vilanova i la } \\
\text { Geltrú }\end{array}$} & \multirow[b]{2}{*}{ TL09B } & \multirow[b]{2}{*}{2} & 1. Vilanova i la Geltrú, S.Pere de Ribes, Sitges, Cubelles, Canyelles, Olivella \\
\hline & & & $\begin{array}{l}\text { 2. Vilafranca del Penedès, Sant Sadurní d'Anoia, Santa Margarida i els Monjos, Gelida, } \\
\text { la Granada, el Vendrell, Calafell. }\end{array}$ \\
\hline Manresa & TL05b & 1 & $\begin{array}{l}\text { Manresa, Berga, Sant Joan de Vilatorrada, Solsona, Sant Vicenç de Castellet, Sallent, } \\
\text { Sant Fruitós de Bages, Súria, Navàs, Santpedor, Navarcles, Cardona, Gironella, Artés, } \\
\text { Puig-reig, Balsareny, Moià. }\end{array}$ \\
\hline Vic & TL08B & 1 & $\begin{array}{l}\text { Vic, Manlleu, Torelló, Sant Pere de Torelló, Tona, Taradell, Roda de Ter, Sant Hipòlit } \\
\text { de Voltregà, Les Masies de Voltregà, Centelles, Lluçà. }\end{array}$ \\
\hline Igualada & TL04B & 1 & $\begin{array}{l}\text { Igualada, Piera, Vilanova del Camí, Santa Margarida de Montbui, Masquefa, } \\
\text { Capellades, Òdena. }\end{array}$ \\
\hline \multirow[b]{2}{*}{ Girona } & \multirow[b]{2}{*}{ TL03GI } & \multirow[b]{2}{*}{2} & 1.Girona, Salt, Cassà de la Selva, Llagostera, Sarrià de Ter, Bescanó, Canet d'Adri. \\
\hline & & & 2.Banyoles, Porqueres \\
\hline \multirow[b]{2}{*}{ Blanes } & \multirow[b]{2}{*}{ TL01GI } & \multirow[b]{2}{*}{2} & 1. Blanes, Lloret de Mar, Tossa de Mar. \\
\hline & & & 2.S.Coloma de Farners, Arbúcies, S.Hilari Sacalm, Anglès, Maçanet de la Selva, Breda \\
\hline Palafrugell & TL05GI & 1 & $\begin{array}{l}\text { Palafrugell, S.Feliu de Guíxols, Palamós, Torroella de Montgrí, La Bisbal d'E., } \\
\text { Calonge, Castell-Platja d'Aro, Begur, Sta.Cristina d'Aro }\end{array}$ \\
\hline Figueres & TL02GI & 1 & Figueres, Roses, Castelló d'Empúries, L’Escala, Vilafant. \\
\hline \multirow[b]{2}{*}{ Olot } & \multirow[b]{2}{*}{ TL04GI } & \multirow[b]{2}{*}{2} & 1.Olot, Sant Joan les Fonts \\
\hline & & & 2.Ripoll, Sant Joan de les Abadesses, Campdevànol \\
\hline Tarragona & TL02T & 1 & Tarragona, Valls, Salou, Vila-seca, Torredembarra, Montblanc, Constantí, Rodonyà \\
\hline \multirow[b]{2}{*}{ Reus } & & & 1. Reus \\
\hline & TL01T & 2 & $\begin{array}{l}\text { 2. Cambrils, Riudoms, Mont-roig del Camp, Vandellòs i l'Hospitalet de l'Infant, la } \\
\text { Selva del Camp, Falset. }\end{array}$ \\
\hline Tortosa & TL03T & 1 & $\begin{array}{l}\text { Tortosa, Amposta, Sant Carles de la Ràpita, Deltebre, Alcanar, Roquetes, Ulldecona, } \\
\text { Godall, Móra d'Ebre, Móra la Nova, Ascó, L’Ametlla de Mar, Gandesa, Horta de Sant } \\
\text { Joan, La Sènia }\end{array}$ \\
\hline Lleida & TL02L & 1 & Lleida, Almacelles, Alcarràs, Alpicat, Almenar, Alfarràs. \\
\hline & & & 1. Balaguer, Mollerussa, les Borges Blanques \\
\hline Balaguer & TL01L & 2 & 2. Tàrrega, Agramunt, Bellpuig, Cervera \\
\hline Seu d'Urgell & TL03L & 1 & La Seu d'Urgell , Puigcerdà, Tremp, La Pobla de Segur, El Pont de Suert, Sort. \\
\hline Vielha e Mijaran & TL04L & 1 & Vielha e Mijaran, Naut Aran, Bossòst. \\
\hline
\end{tabular}

Fuente: PRE/1446/2006, de 9 de mayo, por el cual se da publicidad a los acuerdos de la Comisión de Gobierno de Política Institucional por los cuales se otorga la concesión para la prestación del servicio de TDT-L a los municipios de Cataluña incluidos en las demarcaciones territoriales (DOGC 4637, de 16 de mayo de 2006). 


\section{Estado de los programas públicos de TDT-L en Cataluña (mayo 2010)}

Justo un mes después de finalizar el apagón analógico, en Cataluña solamente emitían 12 de los 37 programas públicos previstos por el Gobierno de la Generalitat. Tres correspondían a programas gestionados por un ayuntamiento en solitario (denominados programas unimunicipales) y nueve a consorcios supramunicipales (ver la Tabla 2).

Los 25 programas públicos que no se habían puesto en marcha se pueden dividir en dos grandes grupos (Ver la Tabla 3). El primero está formado por un conjunto de seis concesionarios que estaban en diferentes fases de desarrollo. El Ayuntamiento de Reus, concesionario del único programa unimunicipal pendiente de encender, tenía previsto iniciar sus emisiones en septiembre de 2010. Reus era el único que no disponía de una televisión analógica propia ${ }^{6}$, de manera que había tenido que invertir en infraestructura y personal. Esto retrasó su puesta en marcha (prevista inicialmente para septiembre de 2009). Los otros tres concesionarios unimunicipales simplemente migraron al digital la emisora que ya explotaban en analógico, de manera que el proceso fue mucho más simple (Guimerà et al., 2009).

Por su parte, el consorcio que agrupa los municipios del Delta del Llobregat tenía previsto iniciar emisiones aunque sin una fecha concreta. Los dos consorcios de la demarcación Blanes estaban avanzando en las negociaciones y en los trámites burocráticos. Los consorcios de la demarcación Reus y Figueres estaban en una situación irregular: como los

Tabla 2. Programas públicos de TDT-L en emisión (mayo de 2010)

\begin{tabular}{|l|c|}
\hline Ente público concesionario de TDT-L & Nombre comercial \\
\hline Ajuntament de Barcelona & Barcelona TV \\
\hline Ajuntament de L'Hospitalet de Llobregat & Televisió de l'Hospitalet \\
\hline Ajuntament de Badalona & Televisió de Badalona \\
\hline $\begin{array}{l}\text { Consorci per a la gestió de la televisió digital local pública de la demarcació } \\
\text { de Granollers (Consorci Teledigital Granollers) }\end{array}$ & Vallès Oriental TV \\
\hline Consorci Teledigital Mollet & Vallès Visió \\
\hline $\begin{array}{l}\text { Consorci per a la gestió de la televisió digital local pública de la demarcació } \\
\text { d'Igualada }\end{array}$ & Conca TV \\
\hline Consorci digital Mataró-Maresme & Maresme Digital TV \\
\hline Consorci Teledigital Maresme Nord & Maresme Digital TV 2 \\
\hline $\begin{array}{l}\text { Consorci per a la gestió de la televisió digital local pública de la } \\
\text { demarcació de Terrassa-Vallès Oest }\end{array}$ & Canal Terrassa Vallès \\
\hline $\begin{array}{l}\text { Consorci per a la gestió de la televisió digital terrestre del Camp de } \\
\text { Tarragona (TACOALT) }\end{array}$ & TAC 12 \\
\hline $\begin{array}{l}\text { Consorci per a la gestió de la televisió digital local pública del Garraf } \\
\text { (Consorci Teledigital Garraf) }\end{array}$ & Canal Blau TV \\
\hline Consorci per a la gestió de la televisió digital local del Penedès & Penedès TV \\
\hline
\end{tabular}

Fuente: elaboración propia a partir de los datos recogidos durante el trabajo de campo y datos facilitados por el CAC. 
ayuntamientos implicados no se ponen de acuerdo, consistorios de la demarcación que disponían de televisión analógica ocupan el programa. Esta situación se debería regularizar, ya que los concesionarios son todos los ayuntamientos consorciados, con lo cual no lo puede explotar uno solo por su cuenta (CAC, 2009).

El segundo grupo lo forman 19 agrupaciones de municipios que no estaban haciendo nada por desarrollar su televisión en mayo de 2010 (ver la Tabla 3). Nueve de ellos reconocieron abiertamente que no pensaban poner en marcha la emisora. Los otros diez, si bien no habían hecho público si renunciaban a gestionar su programa, no estaban desarrollando el proyecto. Destaca el hecho que ocho de estas agrupaciones de municipios ni siquiera habían constituido formalmente el ente de gestión, primer paso administrativo que debían hacer todos los consorcios antes de septiembre de 2006 según la normativa catalana (Guimerà, 2006). De hecho, de los 33 consorcios a crear, en mayo de 2010 sólo se habían constituido 23.

Los datos aportados ponen de manifiesto que el bajo índice de puesta en marcha de canales públicos se debía atribuir a los consorcios, que eran responsables de 24 de los 25 programas pendientes de iniciar emisiones (ver la Tabla 3).

Por el contrario, únicamente nueve de los 33 consorcios previstos estaban emitiendo y sólo cinco más estaban trabajando para ponerse en marcha un mes después del apagón analógico. Es decir, a medio plazo sólo se podía esperar que se pusieran en marcha menos de la mitad. Las siguientes páginas se centran en el análisis de estos consorcios.

\section{La demarcación y el modelo de TDT-L público: dos elementos clave}

Para entender los problemas de implantación de la televisión pública digital local en Cataluña y, en concreto, los consorcios que agrupan diversos municipios, es imprescindible tener en cuenta el modelo de emisora pública que se ha diseñado desde las políticas públicas españolas y catalanas.

Por una parte, la obligación de gestionar conjuntamente un canal que tienen la inmensa mayoría de los ayuntamientos es un primer elemento interpretativo a tener en cuenta y, además, es clave. Por otra, el modelo de emisora pública exigido por la normativa catalana también dificulta el proceso de implantación, ya que es administrativamente complejo y económicamente costoso (Guimerà, 2006).

A partir del Acuerdo de Gobierno ${ }^{7}$ que definía el procedimiento para otorgar las concesiones de TDT a los ayuntamientos, el Gobierno diseñó una televisión de carácter profesional, con la obligación de prestar el servicio de forma continuada y con los recursos humanos y profesionales "adecuados". En diciembre de 2005, la aprobación de la ley 22/2005, de comunicación audiovisual de Cataluña (DOGC 4543, de 3 de enero de 2006) ratificaba el modelo y aún añadía a las obligaciones de los concesionarios (públicos y privados) contribuir al desarrollo de la industria audiovisual catalana (art. 21). 
Tabla 3. Estado de los programas de TDT-L no encendidos (Mayo de 2010)

\begin{tabular}{|c|c|c|}
\hline Ente público concesionario de TDT-L & $\begin{array}{l}\text { Consorcio } \\
\text { constituido }\end{array}$ & Estado del proyecto \\
\hline Ajuntament de Reus & -- & $\begin{array}{l}\text { Emisiones previstas para septiembre de } \\
2010\end{array}$ \\
\hline $\begin{array}{l}\text { Consorci per a la gestió de la televisió digital local pública de la } \\
\text { demarcació Baix Llobregat Delta }\end{array}$ & $\mathrm{Si}$ & En desarrollo \\
\hline $\begin{array}{l}\text { Consorci per a la gestió de la televisió digital local pública de la } \\
\text { demarcació de La Selva Marítima-Canal Blanes }\end{array}$ & $\mathrm{Si}$ & Estudiando opciones \\
\hline $\begin{array}{l}\text { Consorci per a la gestió de la televisió digital local pública de la } \\
\text { demarcació de Blanes }\end{array}$ & No & Estudiando opciones \\
\hline $\begin{array}{l}\text { Consorci per a la gestió de la televisió digital local pública de la } \\
\text { demarcació de l'Alt Empordà }\end{array}$ & No & $\begin{array}{l}\text { Programa ocupado por una televisión } \\
\text { municipal }\end{array}$ \\
\hline Consorci de la televisió digital local del Camp (TDCamp) & $\mathrm{Si}$ & $\begin{array}{l}\text { Programa ocupado por una televisión } \\
\text { municipal }\end{array}$ \\
\hline Consorci per la gestió del servei de TDT local de Lleida & $\mathrm{Si}$ & Parado \\
\hline $\begin{array}{l}\text { Consorci de la televisió local de Sant Adrià de Besòs i Santa } \\
\text { Coloma de Gramenet }\end{array}$ & $\mathrm{Si}$ & Parado (emite carta de ajuste) \\
\hline $\begin{array}{l}\text { Consorci per a la gestió de la televisió digital local pública de la } \\
\text { demarcació de Segarra/Urgell }\end{array}$ & No & Parado \\
\hline $\begin{array}{l}\text { Consorci per a la gestió de la televisió digital local pública del Baix } \\
\text { Llobregat Nord }\end{array}$ & $\mathrm{Si}$ & Parado \\
\hline $\begin{array}{l}\text { Consorci per a la gestió de la televisió digital local pública de la } \\
\text { demarcació de Girona }\end{array}$ & $\mathrm{Si}$ & Parado \\
\hline $\begin{array}{l}\text { Consorci per a la gestió del servei de televisió digital pública de la } \\
\text { demarcació del Bages, Berguedà i Solsonès }\end{array}$ & $\mathrm{Si}$ & Parado \\
\hline Programa 2 demarcació Olot (ente sin nombre oficial) & No & Parado \\
\hline $\begin{array}{l}\text { Consorci per la gestió de la televisió digital local pública del Baix } \\
\text { Empordà }\end{array}$ & No & Parado \\
\hline Consorci de televisió local de les Terres de l'Ebre & $\mathrm{Si}$ & Parado \\
\hline Consorci per a la gestió de la televisió digital local pública d'Aran & $\mathrm{Si}$ & Parado \\
\hline Programa 2 demarcació Balaguer (ente sin nombre oficial) & No & Ha decidido no hacer TV \\
\hline $\begin{array}{l}\text { Consorci per a la gestió de la televisió digital local pública de la } \\
\text { demarcació Baix Llobregat Est }\end{array}$ & No & Ha decidido no hacer TV \\
\hline $\begin{array}{l}\text { Consorci per a la gestió del servei de televisió digital pública del } \\
\text { Baix Llobregat Centre }\end{array}$ & No & Ha decidido no hacer TV \\
\hline Consorci Teledigital Banyoles-Porqueres & $\mathrm{Si}$ & Ha decidido no hacer TV \\
\hline $\begin{array}{l}\text { Consorci per a la gestió de la televisió digital local pública de la } \\
\text { demarcació d'Olot }\end{array}$ & No & Ha decidido no hacer TV \\
\hline $\begin{array}{l}\text { Consorci per a la gestió de la televisió digital local pública de la } \\
\text { demarcació de Sabadell }\end{array}$ & $\mathrm{Si}$ & Ha decidido no hacer TV \\
\hline $\begin{array}{l}\text { Consorci per a la gestió de la televisió local pública demarcació } \\
\text { Vallès Occidental Sud }\end{array}$ & $\mathrm{Si}$ & Ha decidido no hacer TV \\
\hline $\begin{array}{l}\text { Consorci per a la gestió de la televisió digital local pública de la } \\
\text { demarcació La Seu d’Urgell }\end{array}$ & No & Ha decidido no hacer TV \\
\hline $\begin{array}{l}\text { Consorci d'Osona per a la gestió de la televisió digital local pública } \\
\text { canal múltiple de Vic }\end{array}$ & $\mathrm{Si}$ & Ha decidido no hacer TV \\
\hline
\end{tabular}

Fuente: elaboración propia a partir de datos recogidos durante el trabajo de campo y datos facilitados por el Consell de l'Audiovisual de Cataluña en mayo de 2010.

De acuerdo con la ley 41/1995, de televisión local, tanto los prestadores públicos como los privados deben emitir cuatro horas diarias y 32 semanales de "programas televisivos originales" centrados en el territorio para el que emiten. El Gobierno catalán iba más allá y convertía las 32 horas en contenidos de producción propia, ya que dentro de estas horas no se podían contabilizar ni coproducciones con otras televisiones ni los contenidos emitidos en 
cadena. En este sentido, pues, se plantea una televisión con una elevada capacidad de emisión y también de producción (Guimerà, 2006).

En cuanto a la forma de organizar la prestación del servicio, el artículo 33 de la ley 22/2005 describe con bastante detalle y precisión cómo debe hacerse. En primer lugar, el artículo 33.1 determina que las entidades que gestionen las televisiones locales deben dotarse de un "reglamento de organización y funcionamiento" (ROF), que debe definir las misiones de servicio público. Sobre este punto, la ley también establece que la gestión directa del servicio público audiovisual exige que el ente de gestión correspondiente asuma "la definición, la elaboración y distribución de los contenidos audiovisuales, sin perjuicio de la posibilidad de contar con apoyo del sector privado". Así mismo, fija que el nombramiento de los altos cargos debe llevarse a cabo de acuerdo con lo que establezca el ROF. El nombramiento también debe contar con el visto bueno de un consejo de naturaleza asesora y consultiva que ha de evaluar "la capacidad, el mérito y la idoneidad de los candidatos".

Finalmente, el artículo 33.3 determina que "la autonomía de la gestión directa y cotidiana del servicio respecto de los órganos de gobierno correspondientes" se "debe garantizar mediante la suscripción del correspondiente contrato-programa, el cual debe proveer los fondos necesarios para la prestación adecuada del servicio". De este modo, la normativa catalana diseña un sistema que busca separar la gestión más política del canal (correspondiente al consorcio en el caso de las agrupaciones de municipios) de la diaria del programa, que corresponderá al ente que el ROF determine. A la vez, también quiere evitar que la financiación pueda ser una herramienta de presión.

Ciertamente, el modelo exige un amplio desarrollo legal y administrativo que muchos grupos de ayuntamientos no se han visto en condiciones de afrontar por su complejidad intrínseca y por no disponer de recursos humanos ni técnicos para llevarlo a cabo. En este sentido, algunos alcaldes plantearon en las entrevistas la necesidad que la Generalitat pudiera crear ayudas públicas sólo para la creación de todo el aparataje administrativo.

Además, desde que fue formulado, este modelo de televisión se ha asociado a inversiones iniciales elevadas y presupuestos de funcionamiento también altos. Así, a lo largo de todo el trabajo de campo se pudo constatar que todos los representantes de los concesionarios públicos, estuvieran emitiendo o no, coincidan en valorar la TDT-L como "cara".

En las entrevistas se apreció un cierto consenso sobre que la cantidad mínima para crear un programa público en condiciones rondaba el millón de euros. Esta cifra es que la invirtieron el Consorci Digital Mataró-Maresme y el Consorci Teledigital Garraf, los dos primeros en ponerse en marcha en Cataluña (en 2008 y 2009, respectivamente). Desde entonces, esa cifra se tomó como referencia en el sector (CAC, 2009).

En cambio Conca TV, uno de los últimos consorcios en iniciar emisiones en mayo de 2010, había invertido 600.000 euros. A su vez, el consorcio TD Camp contemplaba inversiones que se movían entre los 125.000 y los 600.000 euros. Estas diferencias se explican porque 
los que habían invertido más dinero habían creado nuevos espacios de producción y adquirido nuevo hardware (sobre todo Mataró - Maresme, que empezaba de cero) mientras que las cifras más bajas corresponden a consorcios que trataban de aprovechar al máximo posible las instalaciones que heredaban de antiguas televisiones analógicas de los municipios consorciados (como por ejemplo Igualada o TD Camp).

Las divergencias también llegan a los presupuestos de explotación anual (ver la Tabla 4). Descontado el caso extraordinario de Barcelona $\mathrm{TV}^{8}$, las cifras muestran diferencias de magnitud, hasta el punto que el presupuesto de Badalona puede llegar a ser hasta 20 veces superior al de Maresme Nord ${ }^{9}$.

Tabla 4. Presupuestos de explotación de prestadores públicos de TDT-L (millones de €)

\begin{tabular}{|l|c|c|}
\hline Concesionario & $\mathbf{2 0 0 8}$ & $\mathbf{2 0 0 9}$ \\
\hline Ajuntament de Barcelona & 21,4 & 16,5 \\
\hline Ajuntament de l'Hospitalet de Llobregat & 2,54 & s.d. \\
\hline Ajuntament de Badalona & 2,1 & $3,4^{*}$ \\
\hline $\begin{array}{l}\text { Consorci per a la gestió de la televisió digital pública de la } \\
\text { demarcació de Terrassa - Vallès Oest }\end{array}$ & -- & $1,5^{*}$ \\
\hline Consorci per a la gestió de la televisió digital local del Penedès & -- & 1,06 \\
\hline $\begin{array}{l}\text { Consorci per a la gestió de la televisió digital pública de la } \\
\text { demarcació de Granollers (Consorci Teledigital Granollers) }\end{array}$ & -- & $0,9^{* *}$ \\
\hline Consorci Teledigital Garraf & 0,7 & 1 ’01 \\
\hline Consorci Digital Mataró-Maresme & 0,8 & 0,8 \\
\hline TACOALT & -- & 0,67 \\
\hline Consorci Teledigital Mollet & -- & $0,48^{* *}$ \\
\hline $\begin{array}{l}\text { Consorci per a la gestió de la televisión digital local pública de la } \\
\text { demarcació d'Igualada }\end{array}$ & -- & 0,35 \\
\hline Consorci Teledigital Maresme Nord & -- & 0,18 \\
\hline
\end{tabular}

*Los presupuestos corresponden a empresas de gestión de medios locales que incluyen, además de la televisión, otros soportes como boletines, radios o webs informativas. Por lo tanto, no corresponden sólo a la televisión.

** Presupuesto estimado para 2010 (en 2009 no emitía).

Fuente: elaboración propia a partir de los datos recogidos en el Boletín Oficial de la Provincia de Barcelona y de Tarragona; y los datos facilitados por los directores de las televisiones.

Las diferencias de presupuesto se explican, en parte, por las diferentes ambiciones programáticas o por el hecho de compartir recursos (locales, personal, gastos generales) con otras empresas públicas o con otros medios públicos de los municipios consorciados. En todo caso, las divergencias señalan claramente que los operadores habían encontrado la manera de escalar los recursos económicos necesarios y poner en marcha canales públicos con presupuestos ajustados a sus realidades (CAC, 2009).

\section{El desarrollo de la TDT-L pública: elementos interpretativos}

Sin ninguna duda, la suma de la complejidad del modelo de emisora y la obligación de gestionar conjuntamente un canal por parte de 33 agrupaciones de municipios estaban en la base de la baja implantación de la TDT-L pública en mayo de 2010. Ahora bien, estos dos 
motivos por sí solos no explican quién arrancó y quién no. Es necesario acudir a una amplia serie de factores, combinados entre ellos de maneras diversas, para poder explicar qué ocurrió con los consorcios que no avanzaron y poder entender cuáles son las claves del éxito de los que lo consiguieron antes del apagón analógico.

\subsection{Los consorcios en funcionamiento: rasgos característicos}

El análisis de las características básicas de los nueve consorcios en funcionamiento en el momento estudiado señala que hace falta la combinación de al menos dos de los siguientes cuatro elementos para poder poner en marcha una televisión: el liderazgo dentro del consorcio de un municipio con un mínimo de habitantes; la existencia de cómo mínimo un ayuntamiento con experiencia en televisión analógica; la vinculación de la TDT a un proyecto económico más amplio; o la voluntad política explícita de crear una televisión que contribuya a la vertebración territorial y social de los municipios implicados (ver la Tabla 5).

Tabla 5. Características de los consorcios en emisión (mayo de 2010)

\begin{tabular}{|l|c|c|c|c|}
\hline Consorcio & $\begin{array}{c}\text { Municipio } \\
\text { líder }\end{array}$ & $\begin{array}{c}\text { TV } \\
\text { analógica }\end{array}$ & $\begin{array}{c}\text { Proyecto } \\
\text { económico }\end{array}$ & $\begin{array}{c}\text { Proyecto } \\
\text { territorial }\end{array}$ \\
\hline Consorci Teledigital Granollers & $\begin{array}{c}\text { Mollet del V. } \\
(51.912)\end{array}$ & Sí & No & No \\
\hline Consorci Teledigital Mollet & $\begin{array}{c}\text { Igualada } \\
(38.164)\end{array}$ & Sí & No & Si \\
\hline $\begin{array}{l}\text { Consorci per a la gestió de la televisió digital } \\
\text { local pública de la demarcació d'Igualada }\end{array}$ & $\begin{array}{c}\text { Mataró } \\
(119.780)\end{array}$ & No & Sí & No \\
\hline Consorci digital Mataró-Maresme & $\begin{array}{c}\text { Calella } \\
(18.615)\end{array}$ & Sí & Sí & No \\
\hline Consorci Teledigital Maresme Nord & $\begin{array}{c}\text { Terrassa } \\
(206.245)\end{array}$ & Sí & Sí & No \\
\hline $\begin{array}{l}\text { Consorci per a la gestió de la televisió digital } \\
\text { local pública de la demarcació de Terrassa- } \\
\text { Vallès Oest }\end{array}$ & $\begin{array}{c}\text { Tarragona } \\
(137.536)\end{array}$ & No & No & Si \\
\hline $\begin{array}{l}\text { Consorci per a la gestió de la televisió digital } \\
\text { terrestre del Camp de Tarragona (TACOALT) }\end{array}$ & $\begin{array}{c}\text { Vilanova i la } \\
\text { G. } \\
(64.905)\end{array}$ & Sí & Sí & No \\
\hline Consorci Teledigital Garraf & $\begin{array}{c}\text { Vilafranca del } \\
\text { P. } \\
(37.364)\end{array}$ & Sí & No & No \\
\hline $\begin{array}{l}\text { Consorci per a la gestió de la televisió digital } \\
\text { local del Penedès }\end{array}$ & Padran & \\
\hline
\end{tabular}

Fuente: elaboración propia a partir de datos del Padrón municipal de 2009 facilitados por la Generalitat de Cataluña [En línea]: <http://www.municat.cat> [Consulta: junio de 2010]

\subsubsection{Municipios medianos o grandes con capacidad de liderazgo}

Ocho de los nueve consorcios en marcha en mayo de 2010 estaban liderados por un municipio de más de 35.000 habitantes que había conseguido implicar a todos o buena parte de los municipios más pequeños de su demarcación en el proyecto. La única excepción era el 
consorcio liderado por Calella, con menos de 20.000 habitantes. De todas maneras, era el mayor de todos los que debían crear ese consorcio y lo lideró desde el principio.

Se trata de municipios con potencia económica suficiente para encabezar las inversiones necesarias, tanto materiales como de recursos humanos. También disponen de capacidad para crear un grupo de técnicos que pueda conducir los primeros pasos, hasta que el consorcio cuente con recursos propios para funcionar autónomamente. Así mismo, tienen suficiente peso político para ejercer el liderazgo sin demasiadas discusiones: según la normativa estatal, es el municipio más poblado el que debe liderar el consorcio (Guimerà, Sanmartín y Alborch, 2008).

\subsubsection{Televisiones analógicas profesionalizadas en la base del proyecto}

De los nueve consorcios que emitían, siete estaban liderados por municipios que tenían televisión analógica en funcionamiento. En los casos de Penedès TV (Vendrell TV y Vilafranca TV), Consorci Teledigital Garraf (Maricel TV y Canal Blau TV) y Vallès Oest (Terrassa TV y Matadepera TV), también había una segunda emisora de dimensiones medianas. Sólo los consorcios liderados por Mataró y Tarragona nacieron sin experiencia en televisión, aunque eran dos de las poblaciones líderes más pobladas (ver la Tabla 4).

En mayor o menor grado, las televisiones de los municipios líderes estaban profesionalizadas y tenían una relativamente elevada capacidad de producción. Por tanto, la inmensa mayoría de los consorcios que emitían en mayo de 2010 contaba con una base profesional y de infraestructuras que facilitó la puesta en marcha.

\subsubsection{La vinculación con proyectos estratégicos}

En siete de los nueve consorcios operativos, la TDT-L está vinculada a un proyecto más amplio que se considera estratégico para el desarrollo de la ciudad que lidera el consorcio, bien sea de perfil económico (en cinco casos); o de cohesión territorial (en los dos restantes) (CAC, 2009).

El proyecto Neapolis de Vilanova i la Geltrú quiere integrar en un mismo edificio la televisión pública local, un centro de promoción de la sociedad de la información, la sede en la ciudad de la Universitat Politècnica de Cataluña (UPC); e instalaciones de producción audiovisual de alquiler. El Tecnocampus Mataró Audiovisual, en la capital del Maresme, también pretende crear sinergias entre los dos consorcios de la demarcación y los centros de la UPC en la ciudad, especializados en tecnología del audiovisual (CAC, 2009).

Granollers ha apostado por el proyecto Roca Umbert-Fàbrica de les Arts, que agrupa diversos organismos de producción y promoción cultural de la ciudad, la sede de la televisión pública y también la del Mercat Audiovisual de Cataluña, la mayor feria televisiva de Cataluña y una de las más relevantes del Estado. En Terrassa, la televisión se instaló en el Parc Audiovisual de Catalunya, un clúster con platós de grandes dimensiones y servicios de 
producción. Además, espera crear sinergias con la Escuela Superior de Cine y Audiovisuales de Cataluña (ESCAC, con sede en Terrassa desde 2003) y con la UPC, que ofrece estudios sobre audiovisual y multimedia en la ciudad (CAC, 2009).

Finalmente, en Igualada y en Tarragona existe la voluntad política explícita de estimular la vertebración territorial o social, ya que se percibe alguna carencia en esta línea por parte de las administraciones impulsoras. En el caso de Tarragona se quiso aprovechar la creación de la TDT para establecer una oferta pública inexistente en esa parte de Cataluña, la segunda área metropolitana después de Barcelona. En el caso de Igualada, en 2003 se puso en marcha una televisión analógica como paso previo a la creación de una digital. Uno de sus objetivos, expresado desde el ayuntamiento de Igualada, era contribuir a vertebrar una comarca fuertemente castigada por la desindustrialización desde los años noventa (CAC, 2009).

\subsection{Los consorcios sin televisión: una casuística compleja}

Los consorcios que no habían puesto en marcha la televisión antes del apagón analógico presentan casuísticas muy diversas que combinan factores de naturaleza muy diferente.

\subsubsection{El factor económico: la principal razón}

El más común era el económico: agrupaciones de municipios que dudaban o estaban convencidos que no podían afrontar la inversión que representa crear y gestionar una televisión nueva. Se trataba, mayoritariamente, de consistorios sin televisión analógica. Además, la naturaleza colegial del consorcio hizo que algunos proyectos se parasen porque no todos los municipios implicados garantizaban aportar los fondos que les corresponderían.

El Ayuntamiento de Girona apuntaba en esta línea cuando decidió parar el proyecto, ante el riesgo de tener que asumir la parte de algunos consistorios que no sabían si implicarse. El consorcio del Delta del Llobregat también estuvo parado más de un año a la espera de que todos los municipios vieran clara la sostenibilidad económica del proyecto. Esto, a pesar de disponer en su seno de dos municipios con televisiones analógicas muy potentes (Viladecans y Gavà).

\subsubsection{La falta de liderazgo claro: hacia el estancamiento del consorcio}

Un segundo factor que ralentizó y paralizó la creación de algunos entes de gestión fue la falta de un liderazgo claro en el proyecto y/o la falta de acuerdo en aspectos básicos entre los municipios implicados. Estas situaciones eran muy variadas, pero se podían identificar cuatro tipos básicos:

a) La ausencia del municipio más grande de la demarcación en el liderazgo del consorcio. Las razones aquí eran diversas: falta de interés por hacer televisión, desconocimiento del sector audiovisual que suscita miedos, limitaciones económicas y de recursos humanos disponibles o, simplemente, otras prioridades. Este factor fue clave en la 
demarcación Vic y en los dos consorcios no constituidos de Cornellá y también en el que debería liderar Sabadell.

b) Ante la negativa del o de los municipios grandes a desarrollar la televisión, encabezaban el proceso municipios pequeños con televisión analógica, pero sin suficiente peso político y/o económico para convencer a los más grandes; o para

c) liderarlo solos. El consorcio Baix Nord; con sólo Sant Esteve Sesrovires interesado, y el de Figueres, con la Escala impulsándolo sola, son dos ejemplos. Ambos cuentan con menos de 10.000 habitantes.

d) La incapacidad del municipio líder para implicar al resto. Por las razones que sean, el municipio grande se quedó solo y no consiguió impulsar un ente supramunicipal, que quedó bloqueado por falta de consenso en aspectos básicos. Las causas también son múltiples: escaso interés del resto; falta de acuerdo en aspectos clave como el control político del ente o las aportaciones económicas a hacer por cada municipio; discusiones sobre las sedes que ha de tener la televisión o qué hacer con las instalaciones de las televisiones locales analógicas operativas.

e) La existencia de municipios de dimensiones parecidas y potencialmente capaces de liderar el proyecto estancó las negociaciones. Aquí, la falta de un liderazgo claro dio lugar a conflictos internos que paralizaron el proyecto. Esta situación ha contribuido a bloquear consorcios como el de Vielha e Mijaran.

\subsubsection{El escaso interés por la televisión}

Finalmente, el tercer aspecto es la falta de interés entre los municipios implicados por poner en marcha la televisión local. Este, que se cruzó claramente con el segundo, se tradujo en muchos casos en falta de liderazgo o conflictos internos. Hay tres tipos de razones, bien solas o bien combinadas entre ellas, que ayudan a entender por qué los municipios no tienen interés en la TDT-L pública.

El más habitual, por el número de casos, era la falta de tradición en televisión pública local, cosa que provocó que no se percibiera qué tipo de beneficios puede aportar. Este apareció ligado a la cuestión económica y a la rentabilidad de la inversión, que constituiría la segunda razón. Finalmente, se detectó que los municipios grandes menos interesados en los consorcios públicos eran aquellos que tenían una televisión privada en su demarcación. Éstos consideraban que la emisora privada ya prestaba una cierta función de servicio público. Además, estos ayuntamientos solían tener convenios o contratos con estas televisiones. En este contexto, los gobiernos prefirieron mantener esa relación con la privada y evitar crear una pública, que entendían que podía afectar negativamente a la sostenibilidad de la comercial. 


\subsubsection{La crisis económica como agravante}

El factor económico como elemento para explicar la situación de los operadores públicos fue adquiriendo importancia a medida que avanzaba la crisis económica iniciada en 2008.

Los ayuntamientos vieron como, desde ese año, iban cayendo las recaudaciones de impuestos -sobre todo las relacionadas con la construcción- y cómo iban aumentando las necesidades de los servicios sociales que prestaban en sus municipios. En 2010, las exigencias de recorte del gasto público aprobadas tanto por el Gobierno central como por el autonómico vinieron a ratificar la dificultad de invertir en televisiones, sobre todo por parte de aquellos ayuntamientos sin tradición previa en el audiovisual.

De todas maneras, sería un error atribuir a la crisis económica el estado de la televisión local pública catalana en mayo de 2010. Por un lado, porque cuando se debían crear los consorcios (durante la segunda mitad de 2006), la crisis económica no había hecho, ni mucho menos, su aparición. Así, todo indica que muchas agrupaciones de municipios han decidido no hacer televisión porque no les interesa, independientemente de si pueden afrontar los gastos o no. Por otro lado, porque a mediados de 2010 había agrupaciones de municipios que volvían a intentar la puesta en marcha de la televisión, justo en uno de los momentos más duros de la crisis.

No obstante, no se puede negar que la crisis aportó argumentos a muchos ayuntamientos que ya habían acordado no hacer televisión. En este sentido, uno de los razonamientos que más se oyeron en las entrevistas fue que, si sin la crisis ya no se veía claro hacer televisión, con los problemas económicos aún menos.

\subsubsection{Un exceso de reservas para la iniciativa pública}

Visto desde la perspectiva de 2010, todo indica que la cantidad de programas reservados para la iniciativa pública por parte del Gobierno catalán en 2005 es también un factor clave para entender el bajo porcentaje de canales municipales en marcha. Cinco años después, y visto el comportamiento de los consistorios en este periodo, parece claro que no hacían falta 37 reservas.

De las 11 demarcaciones en las que el ejecutivo catalán reservó más espacio del mínimo marcado por la ley, sólo en cinco se ha ocupado ese espacio extra. Además, en cinco de ellas no se ha puesto en marcha ningún programa público. De todas maneras, este desfase no debe atribuirse al Gobierno autonómico en solitario, sino que los ayuntamientos son también corresponsables. A fin de cuentas, cuando la Generalitat les consultó en 2005 si querían gestionar un canal, sólo uno de los 240 planificados dijo que no. En mayo de 2010, nueve consorcios reconocían explícitamente que no les interesa el medio. 


\section{Conclusiones}

En mayo de 2010, justo un mes después del fin de las emisiones analógicas, la televisión local pública catalana presentaba una situación muy delicada que se podía calificar de crisis del sector: de los 37 programas digitales previstos por la Generalitat de Cataluña, sólo 12 estaban emitiendo en el momento del apagón -apenas un 33\% del total esperado.

Todos los indicios y evidencias recogidos en la investigación apuntan con claridad que esta situación se debía a la manera cómo las políticas públicas de los Gobiernos central y catalán habían gestionado la migración de la televisión local. Dos decisiones del Gobierno central se encuentran en la base del problema. Por un lado, el diseño de la TDT-L a partir de demarcaciones que, en Cataluña, agrupan más de un municipio en todos los casos. Por el otro, el hecho que sólo la mitad del espacio radioeléctrico se puede destinar a la iniciativa pública. La combinación de ambos factores provocó que 33 de los 37 canales públicos previstos los tuvieran que poner en marcha agrupaciones de municipios que, en la gran mayoría de casos, no constituían una unidad política o administrativa previa.

Los consorcios fruto de esas decisiones políticas eran el principal causante de la baja formalización de canales públicos en Cataluña en el momento del apagón analógico. Un primer indicio es que tres de las cuatro televisiones unimunicipales estaban emitiendo y la cuarta lo hizo a corto plazo. Esto indica que para estos actores fue muy fácil ponerse en marcha, ya que no hubo negociaciones ni pactos.

El dato de que sólo nueve de las 33 agrupaciones que debían gestionar un canal público lo habían puesto en marcha en mayo de 2010 aporta un segundo indicio en esta línea. En este sentido, el bajo índice de formalización de entes públicos (12 de 37) se debía atribuir a los consorcios: de los 25 que no emitían a mayo de 2010, 24 correspondían a entes supramunicipales y sólo uno a un ayuntamiento en solitario.

La explicación no se encuentra sólo en el hecho de tener que compartir el espacio radioeléctrico. Un análisis más profundo pone de manifiesto que la mayor parte de los problemas provenían de las dificultades de los consorcios para constituirse y desarrollar el modelo de programa público diseñado por la normativa catalana: complejo desde el punto de vista organizativo; y con una alta capacidad de producción y emisión que necesita fuertes inversiones.

Así, algunos consorcios sufren carencias económicas o estructurales que les impedían poner en marcha la televisión, aunque quisieran. Por otro lado, había quien decía no estar interesado en hacer televisión local y, por tanto, no la pondría en marcha. Finalmente, y lo más importante, los conflictos y desencuentros de todo tipo entre municipios con necesidades, intereses y recursos económicos muy diferentes.

Una idea clave aparece cuando se combinan estos datos: las políticas públicas han diseñado un servicio público audiovisual local tan complejo que se ha revelado de difícil implantación. 
Desde esta perspectiva, el modelo de televisión pública mayoritario en Cataluña, el consorcio, ha sido claramente un freno a la implantación de la TDT-L en el Principado.

La experiencia de los nueve consorcios que sí que habían conseguido arrancar apunta en la misma dirección. Así, son necesarios al menos dos de los cuatro factores que se revelan claves para el éxito. Por una parte, la existencia de un municipio líder con suficiente peso económico y demográfico como para llevar el grueso del proyecto. Por otra parte, que el ayuntamiento líder dispusiera de una televisión municipal analógica, que facilitaba el inicio de emisiones digitales. En tercer lugar, la vinculación de la TDT a un proyecto económico más amplio y estratégico para la ciudad. Finalmente, la voluntad política explícita de crear una televisión que contribuyera a la vertebración territorial y social, sobre todo en zonas con limitaciones en este aspecto.

De los cuatro elementos que ayudan a explicar la situación de los consorcios operativos, los datos apuntan que el más influyente es tener tradición en la gestión de televisión pública analógica: sólo dos de los nueve consorcios que emitían en mayo de 2010 no tenían experiencia en este campo. Ahora bien, la asociación de la TDT-L a proyectos considerados importantes por las administraciones implicadas parece que permite salvar el hándicap aparente que supone no tener una emisora analógica desde la cual partir. De todas maneras, no se puede dejar de lado que las ciudades líderes de los consorcios son en su mayoría grandes y con un cierto peso específico dentro de su territorio, cosa que les permitió liderar el proyecto en su momento.

Ciertamente, la situación de la TDT-L pública en Cataluña en mayo de 2010 es un toque de atención sobre el estado del audiovisual público local en el resto de España. A fin de cuentas la demarcación, la razón primera de la baja implantación de la TDT-L pública, es común a todas las comunidades autónomas. En este sentido, territorios con larga tradición de televisión municipal analógica como Andalucía o con altos porcentajes de programas digitales reservados para los ayuntamientos como Canarias pueden tomar nota de los problemas detectados en Cataluña. En esta comunidad, donde existen una abundante y dilatada experiencia en el sector analógico; y unas políticas decididamente favorables a la TDT-L pública se ha llegado a una situación que sólo se puede calificar de crítica.

\section{Fuentes consultadas}

Badillo, Ángel (2005) [En línea]: "La desregulación invisible: el caso de la televisión local por ondas en España", en EPTIC, Vol. VII, núm. 1, enero -abril, recuperado el 5 de junio de 2010, de http://www.eptic.com.br/arquivos/Revistas/VII,n.1,2005/Badillo.pdf

Consell de l'Audiovisual de Cataluña (CAC) (2009): Diagnòstic de la televisió digital terrestre local a Cataluña (setembre - octubre 2009). Barcelona: CAC

Corominas, Maria (2009): Televisió local a Cataluña. Barcelona: Institut d'Estudis Catalans. 
Fernández Alonso, Isabel y otros (2007): "Políticas de Implantación de la TDT local en España (2005-2006): los casos de las Comunidades Autónomas de Islas Baleares, Madrid, Región de Murcia, Comunidad Valenciana, Galicia, Cataluña y Aragón”, en Observatorio (OBS*) Journal, Vol. 1, Lisboa, OberCom, pp. 205 - 224.

Guimerà i Orts, Josep Àngel (2006): "Local television in Catalonia: a model undergoing an extensive transformation", en Quaderns del CAC, número 26, Barcelona, CAC, pàg. 139 147. Recuperado el 5 de junio de 2010 en http://www.cac.cat/pfw_files/cma/recerca/quaderns_cac/Q26_EN_Guim_ra.pdf

---- y otros (2009): La comunicació local a Cataluña. Informe 2008. Bellaterra: Institut de la Comunicació (InCom) de la Universitat Autònoma de Barcelona (UAB).

----, Sanmartín, Julián y Alborch, Federica (2008): La TDT local pública a Cataluña. Segona fase. Bellaterra: Institut de la Comunicació (InCom) de la Universitat Autònoma de Barcelona (UAB). [Texto mecanografiado]

Marzal, Javier y Casero, Andreu (2008): "La investigación sobre la televisión local en España: nuevas agendas ante el reto de la digitalización", en Zer, Vol. 13 - 25, Leioa, Universidad del País Vasco, pág. 83. -106.

OCL (Observatori de la Comunicació Local) (2005): "La comunicació local", en VV.AA.: Informe de la comunicació a Cataluña 2003-2004 (Eds., M. Corominas; M. de Moragas y J. À. Guimerà). Bellaterra, Castelló de la Plana, Barcelona, València; Servei de Publicacions de la Universitat Autònoma de Barcelona; Publicacions de la Universitat Jaume I; Universitat Pompeu Fabra; Universitat de València. Servei de Publicacions, pàg. 211-230.

Ortega Mohedano, Félix (2009): "La televisión de proximidad en España. Procesos concesionales, análisis de la población y PIB", en RLCS, Revista Latina de Comunicación Social, 64, páginas 385 a 601. La Laguna (Tenerife): Universidad de La Laguna, recuperado el 5 de junio de 2010, de http://www.revistalatinacs.org/09/art/48_847_ULEPICC_10/410rtega.html DOI: 10.4185/RLCS-64-2009-847-585-601

Soriano, Jaume (2007): L'ofici de comunicòleg. Vic: Eumo.

\section{Notas}

[1] Si bien el legislador español había aprobado la ley 41/1995, de 22 de diciembre, de regulación de la televisión local por ondas (BOE 309, de 27 de diciembre) esta ley no entró en vigor hasta una década mas tarde, ya en el contexto de implantación de la TDT. El Gobierno español publicó el plan técnico de televisión local necesario para convocar los concursos en 2004, momento en el cual las primeras comunidades autónomas convocaron los concursos de concesión y, por lo tanto, regularon plenamente las emisoras (Guimerà, 2006). 
[2] En el caso de las demarcaciones que disponen de dos múltiplex, las comunidades autónomas pueden reservar uno entero para cada titularidad. En todo caso, la proporción máxima sigue siendo de 1 a 1 (Guimerà, 2006).

[3] En 2008, vistos los notables problemas y retrasos que sufría la implantación de la TDT local en Cataluña pocos meses antes del apagón analógico, el CAC decidió llevar a cabo una investigación para poder disponer de un diagnóstico propio sobre la transición digital de la televisión local. El CAC encargó al autor de éste artículo el diseño de la investigación, la coordinación y ejecución del trabajo de campo; y la redacción del informe final. El estudio se llevo a cabo bajo la coordinación de Maria Corominas, jefa del Área de Estudios del CAC, quien participó en la redacción del informe final. El CAC (2009) ha publicado una versión ejecutiva del estudio.

[4] El Gobierno del PP aprobó, dos días antes de las elecciones legislativas españolas de 2004, el Real Decreto 439/2004, de 12 de marzo, por el que se aprueba el Plan técnico nacional de televisión digital local (BOE 85, de 4 de abril de 2004). A finales del mismo año, poco después de llegar al Gobierno, el nuevo ejecutivo del PSOE aprobó el Real Decreto 2268/2004, de 3 de diciembre, por el que se modifica el Real Decreto 439/2004, por el que se aprueba el Plan técnico nacional de la televisión digital local (PTN) (BOE 292, de 4 de diciembre de 2004).

[5] La Ley 10/2005, de 14 de junio, de medidas urgentes para el impulso de la televisión digital terrestre, de la liberalización de la televisión por cable y de fomento del pluralismo (BOE 142 de 15 de junio de 2005), establecía la división entre titularidad pública y privada apuntada modificando el artículo 3 de la ley 41/1995.

[6] Desde 1996 hasta 2009, el Ayuntamiento de Reus contó con una emisora (Canal Reus TV) municipal de gestión indirecta explotada por la productora Iniciatives de Televisió SL. La televisión era financiada por el ayuntamiento a través de un contrato - programa trienal. Todos los equipos y instalaciones de la televisión eran de la empresa privada. En el concurso de concesiones de TDT-L privada resuelto el 2006, esta productora obtuvo una concesión para explotar un canal. En septiembre de 2009, la productora empezó a emitir como canal privado y el público desapareció. En consecuencia, el ayuntamiento de Reus debe empezar de cero en la TDT (Guimerà, 2006; CAC, 2009).

[7] Resolución PRE/2804/2005, de 27 de septiembre, por la que se da publicidad al Acuerdo del Gobierno de 20 de septiembre de 2005, por el que se establece el procedimiento de concesión de programas de televisión digital local a los municipios de Cataluña incluidos en las demarcaciones que establece el Plan técnico nacional vigente de la televisión digital local y el régimen jurídico de éstas (DOGC 4482, de 4 de octubre de 2005).

[8] La televisión gestionada por el Ayuntamiento de Barcelona es un fenómeno totalmente excepcional en el contexto de la TDT-L catalana. Incluso sus responsables quisieron 
explicitar en las entrevistas que ellos se sienten "algo más que una televisión local" y esbozaron una definición como "emisora generalista del área metropolitana" de Barcelona, que incluye 4 millones de habitantes. Históricamente, ha sido la mejor dotada de todas las locales catalanas (Guimerà, 2006).

[9] Este caso también es muy particular, ya que el consorcio de Maresme Nord delegó en el de Mataró - Maresme para que le gestionara su programa. De esta manera, Mataró Maresme explota Maresme Digital TV y Maresme Digital TV 2, las dos emisoras públicas de la demarcación. Los 180.000 euros anuales de Maresme Nord son para producir contenidos específicos para Maresme Digital TV 2, mientras que el grueso de la programación es común.

* Este artículo se ha elaborado en el marco del proyecto I+D "Transformaciones del sistema de radio y televisión en España en el contexto de la digitalización (19972010)" financiado por el Ministerio de Educación y Ciencia (ref. SEJ200765109/SOCI), para el periodo 2007-2010. También ha contado con financiación del Consell de l'Audiovisual de Catalunya, en el marco de la investigación "Diagnòstic de la TDT-L a Catalunya 2008 - 2009".

\section{FORMA DE CITAR ESTE TRABAJO EN BIBLIOGRAFÍAS - HOW TO CITE THIS ARTICLE IN BIBLIOGRAPHIES / REFERENCES:}

Guimerá i Orts, J. A., Alborch Gil, F. (2011): "La crisis de la TDT local pública en España: el caso de Cataluña", en Revista Latina de Comunicación Social, 66. La Laguna (Tenerife): Universidad de La Laguna, páginas 292 a 313 recuperado el http://www.revistalatinacs.org/11/art/934_UAB/13_Guimera.html de 2 , de

DOI: $10.4185 / R L C S-66-2011-934-292-313$

Nota: el $\underline{\mathrm{DOI}}$ es parte de la referencia bibliográfica y ha de ir cuando se cite este artículo. 